Article

\title{
Strong and Reversible Adhesion of Interlocked 3D-Microarchitectures
}

\author{
Minho Seong ${ }^{\dagger}$, Hyun-Ha Park ${ }^{\dagger}$, Insol Hwang and Hoon Eui Jeong *(D) \\ Ulsan National Institute of Science and Technology (UNIST), Ulsan 34130, Korea; sung710uio@unist.ac.kr (M.S.); \\ hyunhapark@unist.ac.kr (H.-H.P.); ihwang@unist.ac.kr (I.H.) \\ * Correspondence: hoonejeong@unist.ac.kr; Tel.: +82-52-217-2339 \\ t The authors contributed equally to this work.
}

Received: 13 November 2018; Accepted: 14 January 2019; Published: 15 January 2019

check for updates

\begin{abstract}
Diverse physical interlocking devices have recently been developed based on one-dimensional (1D), high-aspect-ratio inorganic and organic nanomaterials. Although these 1D nanomaterial-based interlocking devices can provide reliable and repeatable shear adhesion, their adhesion in the normal direction is typically very weak. In addition, the high-aspect-ratio, slender structures are mechanically less durable. In this study, we demonstrate a highly flexible and robust interlocking system that exhibits strong and reversible adhesion based on physical interlocking between three-dimensional (3D) microscale architectures. The 3D microstructures have protruding tips on their cylindrical stems, which enable tight mechanical binding between the microstructures. Based on the unique 3D architectures, the interlocking adhesives exhibit remarkable adhesion strengths in both the normal and shear directions. In addition, their adhesion is highly reversible due to the robust mechanical and structural stability of the microstructures. An analytical model is proposed to explain the measured adhesion behavior, which is in good agreement with the experimental results.
\end{abstract}

Keywords: adhesion; flexible adhesive; interlock; interlocking adhesive; reversible adhesion; polyurethane acrylate; $3 \mathrm{D}$ microstructures

\section{Introduction}

In recent years, various fasteners based on a mechanical interlocking between precisely defined architectures have been commercialized (e.g., Microduotec ${ }^{\circledR}$, Gottlieb Binder GmbH \& Co., Germany) and widely utilized for a variety of applications in our daily lives, hospital, and industry. However, these interlocking adhesives are based on macroscopic architectures and thus relatively thick and bulky, which inhibits their integration with diverse smart wearable devices and systems. To this end, various flexible interlocking devices and systems have been developed using one-dimensional (1D), high-aspect-ratio (HAR) nanomaterials such as carbon nanotubes, inorganic nanowires, and polymer nanohairs [1-3]. These 1D nanostructure-based interlocking devices can exhibit reliable and reversible adhesion based on maximized van der Waals interactions between the interlocked nanoscale structures, which enables their application in a broad range of fields [3-11]. For example, Pang et al. developed a highly sensitive mechanical sensor using reversible interlocking of polymeric nanofibers [5]. Liu et al. reported a nanopile interlocking device that can be used for stretchable electrodes and strain sensors [12]. Sun et al. also devised a multifunctional electronic skin based on reversible interlocking of micropillar-wrinkle hybrid structures [13]. Ko et al. utilized Ge/parylene hybrid nanowires to develop a reusable, self-selective connector that exhibits strong shear adhesion in both dry and wet conditions [3]. Oh et al. also reported a mechanical interlocking system that can tightly bind sulfonated poly(arylene ether sulfone) membranes and Nafion catalyst layers in polymer 
electrolyte fuel cells [4]. Unlike conventional bulk and thick interlocking devices, such as Velcro tape, these nanostructure-based miniaturized interlocking devices are not only highly thin and flexible but also can exhibit smart functionalities (e.g., mechanical sensing). Although these 1D nanomaterial-based interlocking devices can provide strong and reversible adhesion in the shear direction, their adhesion in the normal direction is typically very weak [2,3]. Also, the HAR structures are mechanically less durable, which limits their repeatability and reversibility [14].

In this study, we demonstrated a highly flexible and robust reversible interlocking adhesive based on three-dimensional (3D) microscale architectures with protruding tips on their cylindrical stems. When two layers of the microstructure arrays came into contact with each other under external pressure, a microstructural interpenetration and subsequent tight mechanical binding occurred between the mating microstructure layers, which led to strong and repeatable adhesion both in the normal and shear directions without the need for surface modification with chemical moieties. Two different types of polyurethane acrylate (PUA) with different elastic moduli were utilized for the fabrication of the 3D interlocking adhesives to evaluate the effects of the mechanical strengths of the materials on the resulting adhesion behavior. In addition, 3D microstructures with two different tip thicknesses (approximately 5 and $9 \mu \mathrm{m}$ ) were prepared, and their adhesion behavior was investigated. Maximum adhesion strengths of $775 \pm 64 \mathrm{kPa}$ and $447 \pm 71 \mathrm{kPa}$ were obtained in the normal and shear directions, respectively, with PUA interlocking adhesives with $9 \mu \mathrm{m}$-thick tips. Theoretical models were proposed to elucidate the underlying mechanisms of the interlocking adhesion between the 3D microarchitectures.

\section{Materials and Methods}

\subsection{Fabrication of the 3D Microstructures}

First, a Si master with microhole patterns was fabricated using a bilayer of lift-off resist (LOR, Microchem, Westborough, MA, USA) and photoresist (AZ4300, Microchem, USA) [15-17]. The LOR was spin-coated on a dehydrated Si substrate at $1000 \mathrm{rpm}$, followed by thermal baking at $220^{\circ} \mathrm{C}$. To obtain a thick LOR layer, this process was repeated. Then, AZ4300 was spin-coated over the LOR layer $(1000 \mathrm{rpm})$, followed by soft baking at $90{ }^{\circ} \mathrm{C}$. Subsequently, microhole array patterns were formed in the LOR/AZ4300 bilayer by exposing the bilayer to ultraviolet (UV) light $(\lambda=365 \mathrm{~nm}$; dose $=50 \mathrm{~mJ} / \mathrm{cm}^{2}$ ) followed by developing the UV-exposed bilayer with an AZ developer. After rinsing and drying the patterned Si master with deionized water and nitrogen blowing, respectively, a negative replica of the pattern was generated by a replica molding process using a 10:1 (w/w) mixture of polydimethylsiloxane (PDMS) precursor (Sylgard 184, Dow Corning, Midland, MI, USA) and a curing agent. Finally, drops of soft PUA (301RM, Minuta Tech, Osan, Korea) or hard PUA (311RM, Minuta Tech, Korea) were drop-dispensed over the patterned, negative PDMS mold. After uniformly spreading the PUA drops over the PDMS mold by covering the PUA drops with a polyethylene terephthalate (PET) film, the PUA-coated PDMS mold was exposed to UV light $\left(\lambda=365 \mathrm{~nm}\right.$; dose $\left.=200 \mathrm{~mJ} / \mathrm{cm}^{2}\right)$ for photopolymerization of the PUA prepolymer. Subsequent removal of the cured PUA from the PDMS mold resulted in the 3D PUA microstructure array. Finally, the generated 3D PUA microstructure array was exposed to UV light for an additional $3 \mathrm{~h}$ to remove residual polymer radicals and unsaturated acrylates.

\subsection{Scanning Electron Microscope Imaging of the 3D Microstructures}

For scanning electron microscope (SEM) imaging of the 3D microstructures, the fabricated samples were covered with a Pt layer $(3 \mathrm{~nm})$ using a metal sputter. Then, the samples were imaged using a Hitachi S-4800 SEM (Hitachi, Tokyo, Japan). 


\subsection{Confocal Microscope Imaging of the 3D Microstructures}

Confocal microscope images of the 3D microstructures were obtained using a Carl Zeiss LSM 780 confocal microscope (Carl Zeiss, Jena, Germany).

\subsection{Adhesion Measurements}

The adhesion behavior of the interlocked microstructure arrays was examined using custom-built equipment. The equipment had a stage for fixing the samples and parts that could be moved along the horizontal and vertical directions using motors. To measure the normal and shear adhesion strengths, the top and bottom microstructure arrays (size: $100 \mathrm{~mm}^{2}$ ) were brought into contact with a preload of $100 \mathrm{kPa}$ using the equipment. Subsequently, the in-plane strain was applied by the movable parts in the vertical or horizontal directions until separation occurred (retraction rate: $0.5 \mathrm{~mm} / \mathrm{s}$ ). The measurement tests were performed 10 times for each sample. For the cyclic tests, the disassembled microstructure arrays were interlocked again with a preload of $100 \mathrm{kPa}$, followed by the application of the in-plane strain in the vertical or horizontal directions. The interlocking adhesion measurements were repeated during 30 times of repeated assembly and disassembly cycles.

\section{Results}

Figure 1a shows the detailed procedure for the fabrication of the 3D microstructure array. As shown, the 3D microstructure array was prepared by a simple replica molding technique using PUA as a base material. PUA is a UV-curable polymer that can be rapidly cured within a few seconds by UV irradiation [18]. Therefore, various 3D, complicated micro- or nanoscale structures can be simply generated with PUA in a highly precise and rapid manner [15]. Figure $1 b, c$ show the fabricated 3D microstructure array. As shown macroscopically, the generated microstructure array was in the form of a highly flexible thin film (Figure 1b), on which unique 3D microscale architectures were uniformly generated (Figure 1c). The microstructure had unique structural features with protruding tips (Figure 1c,d). The protruding tips enabled robust and effective mechanical interlocking and binding between mating microstructure arrays. We prepared microstructures with two different tip thicknesses of 5 or $9 \mu \mathrm{m}$. The tip diameter, stem diameter, and stem height of the microstructures were approximately 22,11 , and $9 \mu \mathrm{m}$, respectively. The center-to-center pitch of the array was $30 \mu \mathrm{m}$ (Figure 1c).

Figure 2 shows the optical microscope and SEM images of the interlocked microstructures. When two layers of the microstructure array came into contact with each other under the preload, the microstructures interpenetrated each other without a notable misalignment or interlocking failure (Figure 2a). It is presumed that the tapered tip geometry of the microstructure array enabled the successful self-alignment between the mating microstructure arrays by the application of external pressure.

Once the microstructural interpenetration occurred, the protruding tips of the microstructures prevented the disassembly of the interlocked array as the tips of the assembled microstructure arrays overlapped each other. It was noted that previous interlocking devices based on HAR, 1D nanomaterials did not possess such protruding tips or heads. Therefore, their interlocking adhesions were mostly dependent on the van der Waals interactions between the side walls of the interlocked structures, which resulted in limited adhesion performance, especially in the normal direction [2,3]. In contrast to the 1D nanostructure-based interlocking adhesives, our interlocking adhesives had unique $3 \mathrm{D}$ tip configurations. Therefore, the assembled microstructures with protruding 3D tips could exert strong adhesion strengths in both the normal and shear directions. 
a

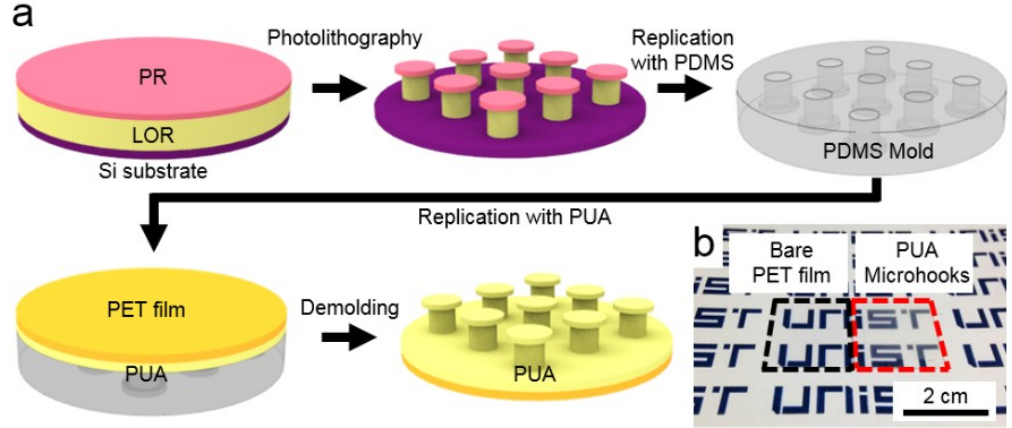

C

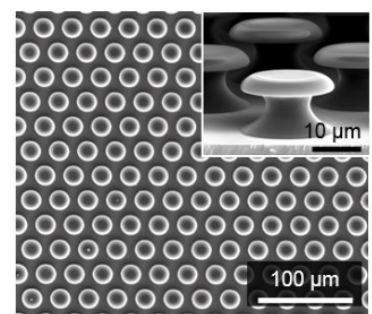

d
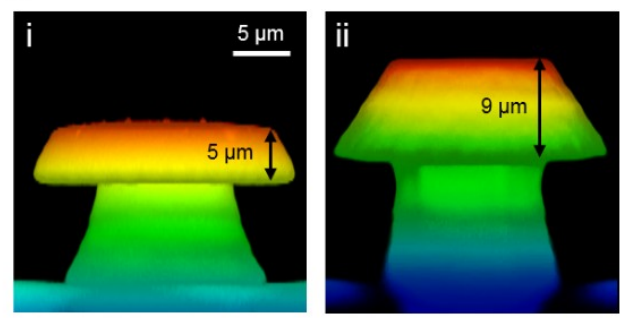

Figure 1. (a) Schematic procedure of the fabrication of the 3D microstructure array; (b) Photograph of the fabricated microstructure array; (c) Top-view and titled scanning electron microscope images of the fabricated microstructure array; (d) Confocal microscope images of the microstructures with different tip thicknesses of (i) $5 \mu \mathrm{m}$ and (ii) $9 \mu \mathrm{m}$.

a

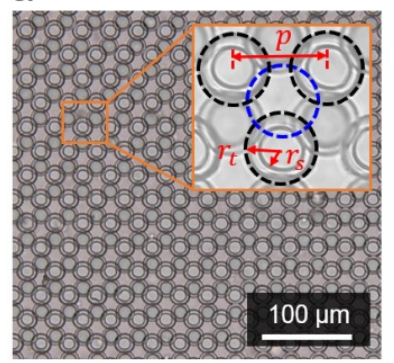

b

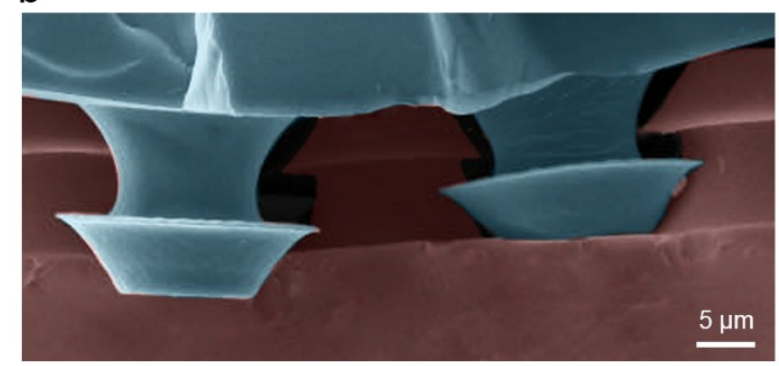

Figure 2. (a) Optical microscope images of the interlocked microstructures; (b) A cross-sectional scanning electron microscope image of the interlocked microstructures.

The adhesion behavior of the interlocked 3D microstructures is displayed in Figure 3. We assumed that the mechanical strength of the tips of the microstructures was the most important factor to determine the adhesion strength and structural stability of the interlocking adhesive. Accordingly, we prepared 3D microstructures with two different tip thicknesses of 5 and $9 \mu \mathrm{m}$. In addition, the microstructures were generated using two different types of PUA (hard PUA and soft PUA). The Young's moduli of the hard PUA and soft PUA were approximately 320 and $20 \mathrm{MPa}$, respectively (Table 1) [19-21]. Figure 3a shows the measured interlocking adhesion strengths of the assembled devices. The averaged adhesion strengths of the interlocked hard PUA microstructures with a tip thickness of $5 \mu \mathrm{m}$ were 101 and $341 \mathrm{kPa}$ in the normal and shear directions, respectively. When the tip thickness increased to $9 \mu \mathrm{m}$, the normal and shear adhesion strengths of the assembled array were further increased to 775 and $447 \mathrm{kPa}$, respectively. This was because the thicker tip had higher mechanical strength than that of the thinner tip, and thus could sustain higher external loads. It is worth noting that the normal adhesion strength of $775 \mathrm{kPa}$ is much higher than those of previously reported interlocking devices based on nanomaterials. For example, the normal adhesion strengths of the $\mathrm{Ge} /$ parylene hybrid nanowire-based adhesive or polymer nanofiber-based interlocking adhesive are negligibly small compared with their shear strengths $[2,3,22]$. In contrast to these nanostructure-based 
interlocking systems, our adhesive could provide strong adhesion strength in both the normal and shear directions. Furthermore, the microstructure array could be fabricated in a highly simple and scalable fashion, while the existing interlocking devices require sophisticated and expensive nanofabrication or synthesis processes. The adhesion strengths of the soft PUA microstructure array were reduced compared with those of the hard PUA microstructures. The averaged normal and shear strengths of the soft PUA microstructure array with a tip thickness of $5 \mu \mathrm{m}$ were 57.0 and $165 \mathrm{kPa}$, respectively. The soft PUA microstructures with a tip thickness of $9 \mu \mathrm{m}$ showed adhesion strengths of 374 and $212 \mathrm{kPa}$ in the normal and shear directions, respectively. The reduced adhesions of the soft PUA microstructures compared with those of the hard PUA microstructures could be readily understood by considering the relatively low elastic modulus of the soft PUA compared to that of the hard PUA. During the cyclic durability tests, the PUA adhesive exhibited a repeatable adhesion without significant loss of adhesion (Figure 3b). It is presumed that the tapered tip geometry of the PUA microstructures (Figure 2b) and flexible PET backing layer of the array enabled the reversible interlocking adhesion without a notable structural failure (Figure 3c).
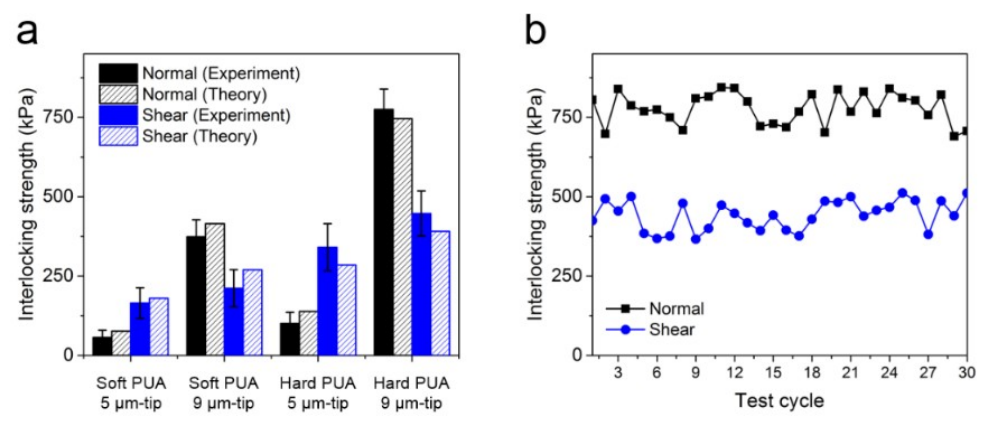

C

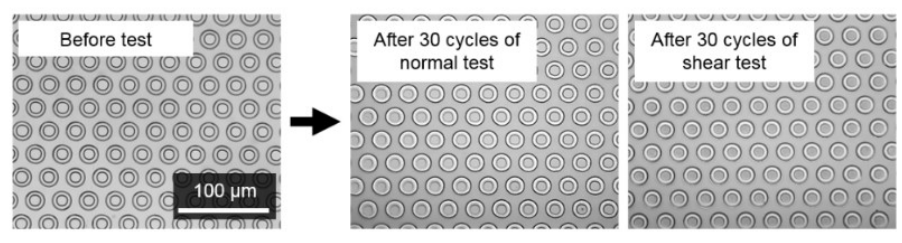

Figure 3. (a) Measured interlocking adhesion strengths of the interlocked soft polyurethane acrylate (PUA) and hard PUA microstructures with different tip thicknesses of $5 \mu \mathrm{m}$ and $9 \mu \mathrm{m}$; (b) Repeatability and durability of the interlocking hard PUA adhesive with a $9 \mu \mathrm{m}$-tip; (c) Optical microscope images of the microstructure array before and after the repeatability tests.

Table 1. Mechanical properties of soft and hard PUA [19-21].

\begin{tabular}{ccc}
\hline PUA & Elastic Modulus (MPa) & Elongation at Break (\%) \\
\hline Soft PUA & 20 & 45 \\
Hard PUA & 320 & 9 \\
\hline
\end{tabular}

To gain further understanding of the interlocking adhesion behavior of the assembled microstructure arrays, a theoretical model was derived by considering the force balance between the interlocked microstructures. For the assembled array, the normal interlocking adhesion force $\left(F_{\text {normal }}\right)$ can be expressed as follows:

$$
F_{\text {normal }}=n F_{\text {ext }}=n\left(F_{\mathrm{b}, \mathrm{t}}+F_{\mathrm{f}, 1}+F_{\mathrm{f}, 2}\right)
$$

where $F_{\text {ext }}$ is the external force acting on the single microstructure, $F_{\mathrm{b}, \mathrm{t}}$ is the bending force acting on the tip, and $F_{\mathrm{f}, 1}$ and $F_{\mathrm{f}, 2}$ are the frictional forces between the side walls of the stem and the tip of 
the interlocked microstructures, respectively (Figure 4a). $n$ is the density of the microstructures. By assuming that the tip is a simple rectangular beam (Figure $4 \mathrm{~b}$ ), $F_{\mathrm{b}, \mathrm{t}}$ can be approximated as follows:

$$
F_{\mathrm{b}, \mathrm{t}}=h_{\mathrm{t}}^{2} \sigma_{\mathrm{u}} \sqrt{\frac{4 r_{\mathrm{t}}^{2}}{3 p^{2}}-\frac{1}{9}}
$$

where $\sigma_{\mathrm{u}}$ is the ultimate tensile strength of the material, $h_{\mathrm{t}}$ is the tip thickness, $r_{\mathrm{t}}$ is the tip radius, and $p$ is the pitch of the array. $F_{\mathrm{f}, 1}$ and $F_{\mathrm{f}, 2}$ are given as follows:

$$
F_{\mathrm{f}, 1}=F_{\mathrm{f}, 2}=F_{\mathrm{f}}=\mu F_{\mathrm{vdw}, \mathrm{cyl}}=\frac{\mu A h_{\mathrm{t}}}{8 \sqrt{2} D_{\mathrm{cyl}}^{5 / 2}} \sqrt{\frac{r_{\mathrm{s}} r_{\mathrm{t}}}{r_{\mathrm{s}}+r_{\mathrm{t}}}}
$$

where $\mu$ is the static coefficient of friction at the interface (approximately 0.1) [23], $F_{\mathrm{vdw}, \mathrm{cyl}}$ is the van der Waals force between the side walls of the stem and the tip of the microstructure, $A$ is the Hamaker constant (approximately $5 \times 10^{-20} \mathrm{~J}$ ) [24], $D_{\text {cyl }}$ is the distance between the two facing side surfaces of the cylinders, and $r_{\mathrm{s}}$ is the stem radius. By applying Equations (2) and (3) in Equation (1), the normal adhesion strength of the interlocked microstructure arrays can be expressed as follows:

$$
F_{\text {normal }}=n\left(F_{\mathrm{b}, \mathrm{t}}+2 \mu F_{\mathrm{vdw}, \mathrm{cyl}}\right)=n h_{\mathrm{t}}^{2} \sigma_{\mathrm{u}} \sqrt{\frac{4 r_{\mathrm{t}}^{2}}{3 p^{2}}-\frac{1}{9}}+\frac{n \mu A h_{\mathrm{t}}}{4 \sqrt{2} D_{\mathrm{cyl}}^{5 / 2}} \sqrt{\frac{r_{\mathrm{s}} r_{\mathrm{t}}}{r_{\mathrm{s}}+r_{\mathrm{t}}}}
$$

For the interlocked microstructures under a normal loading, $D_{\text {cyl }}$ is $\sim 10^{-6} \mathrm{~m}$ (Figure 4a). Therefore, by considering the dominant terms, Equation (4) can be expressed as follows:

$$
F_{\text {normal }} \cong n F_{\mathrm{b}, \mathrm{t}}=n h_{\mathrm{t}}^{2} \sigma_{\mathrm{u}} \sqrt{\frac{4 r_{\mathrm{t}}^{2}}{3 p^{2}}-\frac{1}{9}}
$$

Based on the force balance for the assembled array, the shear adhesion $\left(F_{\text {shear }}\right)$ can also be given as follows (Figure 4c):

$$
F_{\text {shear }}=n F_{\text {ext }} \cos \left(\theta_{\text {ext }}\right)=n\left[F_{\mathrm{x}} \cos \left(\frac{\pi}{2}-\theta_{\mathrm{m}}\right)+F_{\mathrm{y}} \cos \left(\theta_{\mathrm{m}}\right)\right]
$$

where $\theta_{\text {ext }}$ is the angle between $F_{\text {ext }}$ and a horizontal plane and $\theta_{\mathrm{m}}$ is the leaning angle of the microstructure. $F_{\mathrm{x}}$ and $F_{\mathrm{y}}$ are the $\mathrm{x}$ and y components of $F_{\mathrm{ext}}$, respectively, which can be given as follows:

$$
\begin{gathered}
F_{\mathrm{x}}=\frac{1}{3}\left(F_{\mathrm{b}, \mathrm{m}}+F_{\mathrm{f}, 3}\right)=\frac{1}{3}\left(F_{\mathrm{b}, \mathrm{m}}+\mu F_{\mathrm{b}, \mathrm{t}}+\mu F_{\mathrm{vdw}, \mathrm{tip}}\right) \\
F_{\mathrm{y}}=\frac{1}{3}\left(F_{\mathrm{b}, \mathrm{t}}+F_{\mathrm{f}, 1}+F_{\mathrm{f}, 2}\right)=\frac{1}{3}\left(F_{\mathrm{b}, \mathrm{t}}+2 \mu F_{\mathrm{vdw}, \mathrm{cyl}}+\mu F_{\mathrm{b}, \mathrm{m}}\right)
\end{gathered}
$$

where $F_{\mathrm{b}, \mathrm{m}}$ is the bending force acting on the microstructure, $F_{\mathrm{f}, 1-3}$ are the frictional forces at each interface shown in Figure $4 \mathrm{c}$, and $F_{\mathrm{vdw}}$,tip is the van der Waals force between the tip bottoms of the interlocked microstructures. When we assume that the microstructure is a simple cylinder (Figure 4d), $F_{\mathrm{b}, \mathrm{m}}, F_{\mathrm{vdw}, \mathrm{tip}}$, and $F_{\mathrm{vdw}, \mathrm{cyl}}$ can be given by the following equations [24]:

$$
\begin{gathered}
F_{\mathrm{b}, \mathrm{m}}=\frac{\pi\left(\frac{r_{\mathrm{s}}+r_{\mathrm{t}}}{2}\right)^{3}}{4\left(h_{\mathrm{s}}+\frac{h_{\mathrm{t}}}{2}\right)} \sigma_{\mathrm{u}} \\
F_{\mathrm{vdw}, \mathrm{tip}}=\frac{A}{6 \pi D_{\text {tip }}{ }^{3}}\left(2 \theta r_{\mathrm{t}}^{2}-p \sqrt{\frac{r_{\mathrm{t}}^{2}}{3}-\frac{p^{2}}{36}}\right)
\end{gathered}
$$




$$
F_{\mathrm{vdw}, \mathrm{cyl}}=\frac{A h_{\mathrm{t}}}{8 \sqrt{2} D_{\mathrm{cyl}}^{5 / 2}} \sqrt{\frac{r_{\mathrm{s}} r_{\mathrm{t}}}{r_{\mathrm{s}}+r_{\mathrm{t}}}}
$$

where $h_{\mathrm{s}}$ is the stem height, $D_{\text {tip }}$ is the distance between the two facing bottom surfaces of the tips, and $\theta$ is the angle between the horizontal line and the intersecting point formed by the two overlapping circular tips (Figure 4d). Under a shear loading, two adjacent microstructures are tightly interlocked and the facing surfaces of the two interlocked microstructures come into contact. In this case, the inter-surface distances of $D_{\text {cyl }}$ and $D_{\text {tip }}$ can be assumed to be $0.4 \mathrm{~nm}$ (effective separation between two surfaces in contact) [24]. This indicates that $F_{\mathrm{vdw}, \mathrm{tip}}$ and $F_{\mathrm{vdw}, \mathrm{cyl}}$ cannot be neglected for the shear force calculation. By applying Equation (7) in Equation (6), the approximate shear force can be expressed as follows:

$$
F_{\text {shear }}=\frac{n}{3}\left[\left(F_{\mathrm{b}, \mathrm{m}}+\mu F_{\mathrm{b}, \mathrm{t}}+\mu F_{\mathrm{vdw}, \mathrm{tip}}\right) \cos \left(\frac{\pi}{2}-\theta_{\mathrm{m}}\right)+\left(F_{\mathrm{b}, \mathrm{t}}+2 \mu F_{\mathrm{vdw}, \mathrm{cyl}}+\mu F_{\mathrm{b}, \mathrm{m}}\right) \cos \left(\theta_{\mathrm{m}}\right)\right]
$$

By applying Equations (8)-(10) in Equation (11) and using estimated values of each parameter (Table 2), the theoretical shear strengths were calculated. The derived analytical models agreed well with the measurement results (Figure 3a), which showed the validity of our models (see Table 2 for the estimated values of each parameter and force).

a

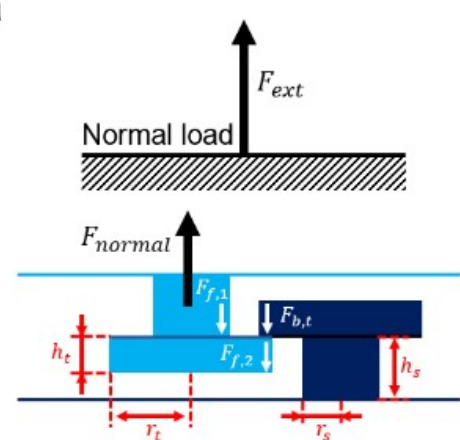

b

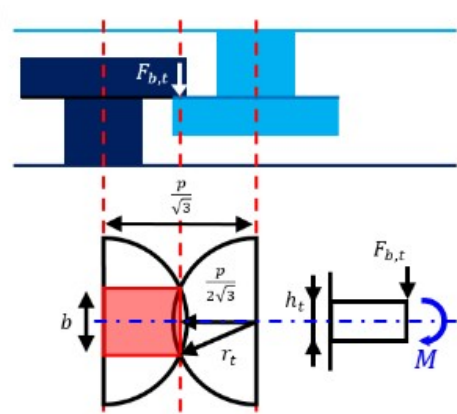

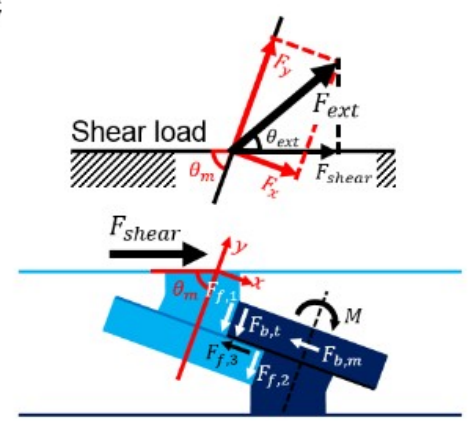

d

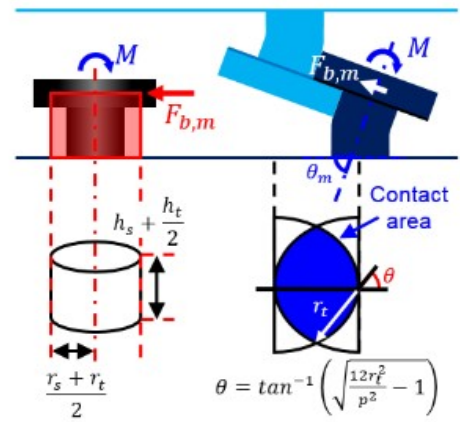

Figure 4. (a) A model system of the interlocked microstructures under a normal loading; (b) A schematic illustration describing the geometry of the microstructure and the bending force acting on the tip of the microstructures $\left(F_{\mathrm{b}, t}\right)$; (c) A model system of the interlocked microstructures under a shear loading; (d) A schematic illustration describing the geometry of the microstructures and the bending force acting on the microstructures $\left(F_{\mathrm{b}, \mathrm{m}}\right)$. 
Table 2. Estimated values of each parameter and force used for the theoretical calculation of the interlocking adhesion.

\begin{tabular}{cccccc}
\hline \multirow{2}{*}{ Parameters } & \multicolumn{2}{c}{ Values } & \multirow{2}{*}{ Forces } & \multicolumn{2}{c}{ Values $[\mu \mathrm{N}]$} \\
\cline { 2 - 3 } \cline { 5 - 6 } & Thin-Tip & Thick-Tip & & $\boldsymbol{F}_{\text {normal }}$ & $F_{\text {shear }}$ \\
\hline$h_{\mathrm{t}}$ & $5 \mu \mathrm{m}$ & $9 \mu \mathrm{m}$ & $F_{\mathrm{b}, \mathrm{t}}$ & $4 \times 10-4 \times 10^{2}$ & $7 \times 10-4 \times 10^{2}$ \\
$r_{\mathrm{t}}$ & $10 \mu \mathrm{m}$ & $12 \mu \mathrm{m}$ & $\mu F_{\mathrm{vdw}, \mathrm{cyl}}$ & $\sim 0\left(<10^{-9}\right)$ & $1-2$ \\
$h_{\mathrm{s}}$ & $8 \mu \mathrm{m}$ & $10 \mu \mathrm{m}$ & $F_{\mathrm{b}, \mathrm{m}}$ & - & $3 \times 10^{2}-6 \times 10^{2}$ \\
$r_{\mathrm{s}}$ & $5 \mu \mathrm{m}$ & $6 \mu \mathrm{m}$ & $\mu F_{\mathrm{vdw}, \mathrm{tip}}$ & - & $2 \times 10^{2}-3 \times 10^{2}$ \\
$p$ & & $30 \mu \mathrm{m}$ & - & - & - \\
$n$ & $2 \times 10^{5} \mathrm{~cm}^{-2}$ & - & - & - \\
$\mu[23]$ & $1 \times 10^{-1}$ & - & - & - \\
$A[24]$ & \multicolumn{2}{c}{$5 \times 10^{-20} \mathrm{~J}$} & - & - & - \\
\hline
\end{tabular}

\section{Conclusions}

In summary, a highly flexible and reversible thin film adhesive was demonstrated based on a reversible interlocking between precisely defined microstructures. Unlike previously reported HAR, 1D nanomaterial-based interlocking systems, our interlocking device utilized unique 3D microscale architectures with protruding tips. Accordingly, the interlocking adhesives could not only exhibit strong normal and shear adhesion strengths but also secure robust structural stability and repeatability. We also showed that the adhesion strengths could be elaborated by increasing the tip thickness of the microstructures or by using materials with high elastic modulus. Maximum interlocking adhesions of $775 \pm 64 \mathrm{kPa}$ and $447 \pm 71 \mathrm{kPa}$ were obtained in the normal and shear directions, respectively, using the assembled hard PUA microstructure array with a tip thickness of $9 \mu \mathrm{m}$. By integrating various functional nanomaterials into the microstructures, this 3D microarchitecture-based reversible interlocking adhesive should contribute to the development of advanced flexible electronic, mechanical, and biomedical devices.

Author Contributions: Conceptualization, M.S., H.-H.P. and H.E.J.; Methodology, M.S., H.-H.P., I.H. and H.E.J.; Experiments, M.S., and H.-H.P.; Analysis, M.S., H.-H.P., I.H. and H.E.J.; Writing and Editing, M.S., H.-H.P., I.H. and H.E.J.; Supervision, H.E.J.; Funding Acquisition, H.E.J.

Funding: This work was supported by the Institute for Information \& communications Technology Promotion (IITP) grant funded by the Korea government (MSIP) (Nos. 2017000910001100, 20180007560011001).

Conflicts of Interest: The authors declare no conflict of interest.

\section{References}

1. Brubaker, C.E.; Messersmith, P.B. The present and future of biologically inspired adhesive interfaces and materials. Langmuir 2012, 28, 2200-2205. [CrossRef] [PubMed]

2. Pang, C.; Kim, T.I.; Bae, W.G.; Kang, D.; Kim, S.M.; Suh, K.Y. Bioinspired reversible interlocker using regularly arrayed high aspect-ratio polymer fibers. Adv. Mater. 2012, 24, 475-479. [CrossRef] [PubMed]

3. Ko, H.; Zhang, Z.X.; Chueh, Y.L.; Ho, J.C.; Lee, J.; Fearing, R.S.; Javey, A. Wet and dry adhesion properties of self-selective nanowire connectors. Adv. Funct. Mater. 2009, 19, 3098-3102. [CrossRef]

4. Oh, K.H.; Kang, H.S.; Choo, M.J.; Jang, D.H.; Lee, D.; Lee, D.G.; Kim, T.H.; Hong, Y.T.; Park, J.K.; Kim, H.T. Interlocking membrane/catalyst layer interface for high mechanical robustness of hydrocarbon-membrane-based polymer electrolyte membrane fuel cells. Adv. Mater. 2015, 27, 2974-2980. [CrossRef] [PubMed]

5. Pang, C.; Lee, G.Y.; Kim, T.I.; Kim, S.M.; Kim, H.N.; Ahn, S.H.; Suh, K.Y. A flexible and highly sensitive strain-gauge sensor using reversible interlocking of nanofibres. Nat. Mater. 2012, 11, 795-801. [CrossRef]

6. Lee, C.H.; Kim, D.R.; Zheng, X.L. Fabrication of nanowire electronics on nonconventional substrates by water-assisted transfer printing method. Nano Lett. 2011, 11, 3435-3439. [CrossRef]

7. Yuk, S.; Choo, M.J.; Lee, D.; Guim, H.; Kim, T.H.; Lee, D.G.; Choi, S.; Lee, D.H.; Doo, G.; Hong, Y.T.; et al. Three-dimensional interlocking interface: Mechanical nanofastener for high interfacial robustness of polymer electrolyte membrane fuel cells. Adv. Mater. 2017, 29, 1603056. [CrossRef] 
8. Gorb, S.N. Biological attachment devices: Exploring nature's diversity for biomimetics. Philos. Trans. R. Soc. A 2008, 366, 1557-1574. [CrossRef]

9. Chen, C.M.; Chiang, C.L.; Lai, C.L.; Xie, T.; Yang, S. Buckling-based strong dry adhesives via interlocking. Adv. Funct. Mater. 2013, 23, 3813-3823. [CrossRef]

10. Yang, S.Y.; O'Cearbhaill, E.D.; Sisk, G.C.; Park, K.M.; Cho, W.K.; Villiger, M.; Bouma, B.E.; Pomahac, B.; Karp, J.M. A bio-inspired swellable microneedle adhesive for mechanical interlocking with tissue. Nat. Commun. 2013, 4, 1702. [CrossRef]

11. Fischer, S.C.L.; Gross, K.; Abad, O.T.; Becker, M.M.; Park, E.; Hensel, R.; Arzt, E. Funnel-shaped microstructures for strong reversible adhesion. Adv. Mater. Interfaces 2017, 4, 1700292. [CrossRef]

12. Liu, Z.Y.; Wang, X.T.; Qi, D.P.; Xu, C.; Yu, J.C.; Liu, Y.Q.; Jiang, Y.; Liedberg, B.; Chen, X.D. High-adhesion stretchable electrodes based on nanopile interlocking. Adv. Mater. 2017, 29, 1603382. [CrossRef] [PubMed]

13. Sun, K.; Ko, H.; Park, H.-H.; Seong, M.; Lee, S.-H.; Yi, H.; Park, H.W.; Kim, T.-I.; Pang, C.; Jeong, H.E. Hybrid architectures of heterogeneous carbon nanotube composite microstructures enable multiaxial strain perception with high sensitivity and ultrabroad sensing range. Small 2018, 14, 1803411. [CrossRef] [PubMed]

14. Kim, T.I.; Jeong, H.E.; Suh, K.Y.; Lee, H.H. Stooped nanohairs: Geometry-controllable, unidirectional, reversible, and robust gecko-like dry adhesive. Adv. Mater. 2009, 21, 2276-2281. [CrossRef]

15. Park, H.H.; Seong, M.; Sun, K.; Ko, H.; Kim, S.M.; Jeong, H.E. Flexible and Shape-Reconfigurable Hydrogel Interlocking Adhesives for High Adhesion in Wet Environments Based on Anisotropic Swelling of Hydrogel Microstructures. ACS Macro Lett. 2017, 6, 1325-1330. [CrossRef]

16. Yi, H.; Seong, M.; Sun, K.; Hwang, I.; Lee, K.; Cha, C.; Kim, T.I.; Jeong, H.E. Wet-responsive, reconfigurable, and biocompatible hydrogel adhesive films for transfer printing of nanomembranes. Adv. Funct. Mater. 2018, 28, 1706498. [CrossRef]

17. Yi, H.; Kang, M.; Kwak, M.K.; Jeong, H.E. Simple and reliable fabrication of bioinspired mushroom-shaped micropillars with precisely controlled tip geometries. ACS Appl. Mater. Interfaces 2016, 8, 22671-22678. [CrossRef] [PubMed]

18. Kwak, R.; Park, H.H.; Ko, H.; Seong, M.; Kwak, M.K.; Jeong, H.E. Partially cured photopolymer with gradient bingham plastic behaviors as a versatile deformable material. ACS Macro Lett. 2017, 6, 561-565. [CrossRef]

19. Choi, S.J.; Kim, H.N.; Bae, W.G.; Suh, K.Y. Modulus- and surface energy-tunable ultraviolet-curable polyurethane acrylate: Properties and applications. J. Mater. Chem. 2011, 21, 14325-14335. [CrossRef]

20. Yi, H.; Hwang, I.; Lee, J.H.; Lee, D.; Lim, H.; Tahk, D.; Sung, M.; Bae, W.G.; Choi, S.J.; Kwak, M.K.; et al. Continuous and scalable fabrication of bioinspired dry adhesives via a roll-to-roll process with modulated ultraviolet-curable resin. ACS Appl. Mater. Interfaces 2014, 6, 14590-14599. [CrossRef]

21. Seong, M.; Jeong, C.; Yi, H.; Park, H.H.; Bae, W.G.; Park, Y.B.; Jeong, H.E. Adhesion of bioinspired nanocomposite microstructure at high temperatures. Appl. Surf. Sci. 2017, 413, 275-283. [CrossRef]

22. Pang, C.; Kwak, M.K.; Lee, C.; Jeong, H.E.; Bae, W.G.; Suh, K.Y. Nano meets beetles from wing to tiptoe: Versatile tools for smart and reversible adhesions. Nano Today 2012, 7, 496-513. [CrossRef]

23. Alahmadi, A. Influence of triboelectrification on friction coefficient. Int. J. Sci. Eng. Res. 2014, 5, $22-29$.

24. Leckband, D.; Israelachvili, J. Intermolecular forces in biology. Q. Rev. Biophys. 2001, 34, 105-267. [CrossRef] [PubMed]

(C) 2019 by the authors. Licensee MDPI, Basel, Switzerland. This article is an open access article distributed under the terms and conditions of the Creative Commons Attribution (CC BY) license (http://creativecommons.org/licenses/by/4.0/). 